\title{
Short-term safety and effectiveness of tension-free vaginal tape (TVT) sling insertion for urodynamic stress incontinence alongside other gynaecological operations
}

\author{
Sharif I. M. F. Ismail • Seumas Eckford
}

Received: 21 July 2010 / Accepted: 10 March 2011 /Published online: 8 April 2011

(C) Springer-Verlag 2011

\begin{abstract}
The aim of this study was to assess the safety, effectiveness and pattern of inserting tension-free vaginal tape (TVT) slings alongside other gynaecological operations. It was a retrospective case note review of 116 patients who had a range of minor and major vaginal, laparoscopic and abdominal procedures at the same time as having TVT sling insertion at five district general hospitals in the UK. It showed an overall incidence of complications of $22.4 \%$ and laparotomy was not required. A patient had bladder
\end{abstract}

S. I. M. F. Ismail

Department of Obstetrics and Gynaecology, Singleton Hospital,

Swansea, South Wales, UK

\section{S. I. M. F. Ismail}

Department of Obstetrics and Gynaecology,

Wrexham Maelor Hospital,

Wrexham, North Wales, UK

S. I. M. F. Ismail

Department of Obstetrics and Gynaecology,

Royal Bournemouth Hospital,

Dorset, UK

S. I. M. F. Ismail $\cdot$ S. Eckford

Department of Obstetrics and Gynaecology,

North Devon District Hospital,

Barnstaple,

Devon, UK

\section{S. I. M. F. Ismail}

Department of Obstetrics and Gynaecology,

Yeovil District Hospital,

Somerset, UK

\section{S. I. M. F. Ismail ( $₫)$}

Department of Obstetrics and Gynaecology,

Royal Sussex County Hospital,

Brighton BN2 5BE, UK

e-mail: sharif212121@yahoo.co.uk perforation, another had blood transfusion, a third had infection at the site of needle passing, and three patients had urinary tract infection. The overall incidence of complications in this series was significantly higher in those having major concomitant surgery than in the total group. Although $9.5 \%$ of patients had catheters for more than a week, only two patients $(1.7 \%)$ required prolonged intermittent self-catheterisation. Development of overactive bladder symptoms was reported by $8.5 \%$ of those who had pure urodynamic stress incontinence prior to surgery. Ninety-four per cent of patients were dry at 6 weeks. All major abdominal procedures were carried out under general anaesthesia prior to TVT sling insertion. A considerable variation was observed in operative and anaesthetic technique with all other concomitant procedures. These results demonstrated that TVT sling insertion alongside other minor and major gynaecological procedures did not undermine the safety or effectiveness of TVT sling insertion. Operative and anaesthetic practice varied considerably, calling for randomised controlled trials to provide evidence for best practice.

Keywords Concomitant surgery - Effectiveness · Safety · Short term · Subjective $\cdot$ Tension-free vaginal tape (TVT) sling

\section{Background}

Patients having tension-free vaginal tape (TVT) sling insertion for urodynamic stress incontinence may also have other gynaecological condition(s) that necessitate additional gynaecological surgery. This particularly applies to pelvic organ prolapse as there is a recognised association between the two conditions [1]. Less commonly, patients may have 
other problems that require surgical management, such as abdominal hysterectomy for heavy periods [2]. In such situations, there is a choice between performing both procedures at the same time or in two separate settings. Although there are published reports on TVT sling insertions alongside other gynaecological procedures, all of these reports are limited to vaginal management of pelvic organ prolapse and vaginal or laparoscopic-assisted vaginal hysterectomy [3-14]. Of these studies, one looked at prophylactic insertion of TVT sling insertion for occult stress incontinence at the same time as having vaginal surgery for severe (grade 3 or more) pelvic organ prolapse [3] and two included patients with urodynamic as well as occult stress incontinence [10,13].

This limitation was highlighted by the National Institute of Clinical Excellence in its guidance on the use of the TVT sling [15]. This means that clinicians do not have clear guidance as to how best to manage urodynamic stress incontinence of urine in the era of mid-urethral tape slings at the same time as dealing with other gynaecological problems. It also means that there is no account of current practice under these circumstances. The aim of this study was to look at the safety and short-term effectiveness of TVT sling insertions alongside other gynaecological procedures and provide an account on how such concomitant procedures are carried out.

\section{Method}

The primary outcome measure for this study was the incidence of operative or immediate postoperative complications, including voiding dysfunction, during and/or following TVT sling insertion alongside other gynaecological procedures. Subjective outcome at the 6-week follow-up visit was considered as the secondary outcome measure. The nature and order of concomitant gynaecological surgery, type of anaesthesia used and hospital stay were noted.

Sample size calculation was based on noting 39\% complication rate when TVT sling insertion was performed alone [16]. To detect a $20 \%$ increase in this rate to $59 \%$, with $80 \%$ power and $95 \%$ confidence ( 0.05 significance), required 107 cases.

In view of the limited number of such procedures carried out in individual hospitals, it was necessary to get cases from several hospitals, and it was possible to obtain data from five placements. Data were collected from patient notes using data collection sheets and downloaded onto Microsoft Works 5.0 for Windows database (www.msn. com). Statistical tests were carried out on Stata 6.0 for Windows (www.stata.com). Normally distributed continuous data were described using the mean and standard deviation, resorting to the median and interquartile range for those not fitting a normal distribution. Categorical data were described using numbers and percentage and compared using $\chi^{2}$ test.

Insertions were made according to the technique described by Ulmsten et al. [17]. Patients were followed up at 6 weeks and outcome was assessed subjectively, cure being defined as no leakage, improvement when there was significant reduction in leakage such that patients did not need further management and failure when there was inadequate improvement. The development of overactive bladder symptoms in those with pure urodynamic stress incontinence was noted.

\section{Findings}

A total of 116 patients were identified from theatre information systems in the five hospitals since the start of using the TVT sling in 1999 until 2007, and all the notes were available. The number of patients included from each hospital is shown in Table 1. Patients' background features are shown in Table 2. Operations were performed by different consultants or under their direct supervision. Two cases had a combination of general and regional anaesthesia: One had spinal until the TVT sling was inserted and the other was woken up after completion of vaginal hysterectomy and posterior repair for cough test adjustment of the TVT tension. The nature of concomitant surgery is shown in Table 3 and the order in which surgery was performed shown in Table 4. Operative and postoperative complications are shown in Table 5. Subjective outcome at 6 weeks of follow-up is shown in Table 6.

\section{Discussion}

This study looked at concomitant gynaecological surgery alongside TVT sling insertion, taking complications as its primary outcome measure. The overall incidence of complications in this study was $22.4 \%$. There was one bladder perforation and no one required laparotomy, which is better than encountered in the randomised trial that

Table 1 Number of patients from individual hospitals

\begin{tabular}{ll}
\hline Hospital & Number (\%) \\
\hline Singleton Hospital & $15(12.9)$ \\
Wrexham Maelor Hospital & $14(12.1)$ \\
North Devon District Hospital & $21(18.1)$ \\
Royal Bournemouth Hospital & $35(30.2)$ \\
Yeovil District Hospital & $31(26.7)$ \\
Total & 116 \\
\hline
\end{tabular}


Table 2 Background features

\begin{tabular}{lll}
\hline Feature & Statistical test & Result \\
\hline Age (years) & Mean \pm SD & $57.3 \pm 12.8$ \\
Previous hysterectomy & Number (\%) & $41(35.3)$ \\
Previous continence surgery & Number (\%) & $13(11.2)$ \\
Previous prolapse surgery & Number (\%) & $23(19.8)$ \\
Medical problems & Number (\%) & $59(50.9)$ \\
Cardiopulmonary & & $33(28.4)$ \\
Bone and joint & & $16(13.8)$ \\
Mixed incontinence (urodynamic) & Number (\%) & $10(8.6)$ \\
Type of anaesthesia & Number (\%) & \\
Spinal/epidural & & $68(58.6)$ \\
General & & $46(39.7)$ \\
Combination & & $2(1.7)$ \\
Postoperative stay (days) & Median (interquartile range) & $3(2-5)$ \\
\hline
\end{tabular}

compared TVT to Burch colposuspension [16]. One patient required blood transfusion and another had infection at the site of passing the needle holding the TVT sling. Urinary tract infection developed in three cases $(2.6 \%)$. Whilst these are less than that reported in the randomised controlled trial which compared TVT sling to Burch colposuspension [16], one must bear in mind that prospective studies may have better recording of complications.

The low incidence of complications in this series matches most of the published studies $[3,4,7-9,12,13]$. On the other hand, one study [5] showed an increased incidence of bladder perforation in those having sling insertion alongside vaginal hysterectomy, mostly in those with previous continence surgery. Another study reported

Table 3 Type of concomitant procedures

\begin{tabular}{lrc}
\hline Type of concomitant procedure & Number $^{\mathrm{a}}$ & Percentage \\
\hline Minor surgery & 37 & 31.9 \\
Hysteroscopy & 6 & 5.2 \\
Mirena insertion/change & 9 & 7.8 \\
Laparoscopic sterilisation & 6 & 5.2 \\
Bladder biopsy & 2 & 1.7 \\
Vulval procedure & 9 & 7.8 \\
Major vaginal surgery & 75 & 64.7 \\
Anterior repair & 28 & 24.1 \\
Posterior repair & 33 & 28.4 \\
Anterior and posterior repair & 14 & 12.1 \\
Sacrospinous fixation & 1 & 0.9 \\
Vaginal hysterectomy & 12 & 10.3 \\
Major abdominal surgery & 5 & 4.3 \\
Hysterectomy & 4 & 3.4 \\
Salpingo-oophorectomy & 4 & 3.4 \\
Sacrocolpopexy & 1 & 0.9 \\
\hline
\end{tabular}

${ }^{\mathrm{a}}$ Some patients had more than one concomitant procedure significantly more blood loss in patients having vaginal surgery for pelvic organ prolapse alongside TVT sling insertion in comparison to those having TVT sling insertion alone [11]. However, the study did not indicate whether this was the result of TVT sling insertion or surgery for pelvic organ prolapse. A bladder perforation rate of $6.7 \%$ was reported by one study [9] which included 45 patients, of whom eight had sling insertion alone, and the results were grouped together, making it difficult to establish the incidence in patients having concomitant surgery. Another one, which only involved surgery for pelvic organ prolapse, reported a complication rate of $29.7 \%$ [6]. This study, however, grouped all complications, including anaesthetic ones, together without providing much detail, making it difficult to make a proper comparison of individual complications.

The incidence of complications in this series was significantly higher in those having major concomitant surgery than in the total group. However, this applied only to all complications grouped together rather than individual complications. The rate was still lower than the rate of complications encountered in the randomised controlled trial that compared TVT sling to Burch colposuspension [16]. Whilst it is logical that major surgery would be associated with more incidence of complications than minor surgery, a similar observation was not reported by any of the published studies that looked at TVT sling insertion alongside other gynaecological procedures [4-14]. Nonetheless, published studies looked at a much smaller range of concomitant gynaecological procedures, whereas this series included the full range of concomitant gynaecological operations. The rate was not significantly higher in those who had previous continence surgery. It is important to bear in mind that neither this series nor the published studies were powered to compare the safety of concomitant surgery in different subgroups of patients. 
Table 4 Order of performing concomitant gynaecological surgery

\begin{tabular}{lccc}
\hline Type of concomitant surgery & Number & Before TVT & After TVT \\
\hline Laparoscopic surgery & 8 & $6(75.0 \%)$ & $2(25.0 \%)$ \\
Major abdominal surgery & 5 & $4(80.0 \%)$ & $1(20.0 \%)$ \\
Vaginal hysterectomy & 12 & $12(100.0 \%)$ & $0(0.0 \%)$ \\
Anterior repair & 42 & $29(69.0 \%)$ & $13(31.0 \%)$ \\
Posterior repair & 47 & $24(51.1 \%)$ & $23(48.9 \%)$ \\
Minor vaginal surgery & 30 & $11(36.7 \%)$ & $19(63.3 \%)$ \\
\hline
\end{tabular}

The link between bladder perforation and previous continence surgery is controversial. For TVT sling insertion without concomitant surgery, one study showed a significant association [18], though another did not [19]. The patient who had bladder perforation in this series did not have previous continence surgery and did not have a major concomitant gynaecological operation. An association was noted in one of the studies that compared TVT sling insertion with and without vaginal hysterectomy [5]. Two studies reported at least half their bladder perforations in patients with prior continence surgery during TVT sling insertion alongside vaginal surgery for pelvic organ prolapse $[4,11]$ as well as without concomitant surgery [12]. It is important to bear in mind that none of these studies, including the series outlined here, was powered to detect an association between previous continence surgery and bladder perforation.

Almost a tenth of patients had catheters for more than a week, which is higher than reported in the randomised trial [16]. On the other hand, only two patients $(1.7 \%)$ required intermittent self-catheterisation for months, which is lower than in the randomised trial [16]. The different ways of bladder management in published studies precludes direct comparison. No catheters were inserted in one study [4] and all the catheters were removed within 9 days in another [9]. Three non-comparative studies showed a higher incidence of voiding dysfunction than encountered in this series. In the first one [8], where no catheter was left more than 5 days irrespective of the postvoid residual volume, five patients $(9.1 \%)$ required intermittent self-catheterisation. In the second one [13], where suprapubic catheters were checked for residual urine volume after $72 \mathrm{~h}$ before removal, $12 \%$ of patients had dysfunctional voiding and $3 \%$ of patients required clean intermittent self-catheterisation for up to 3 weeks postoperatively, which is higher than in this series. In the third [6], 43\% of patients required catheterisation more than 3 days, which is more than that encountered in this series. The author noted an association between voiding dysfunction and concomitant posterior vaginal wall repair as well as previous continence surgery. On the other hand, $6.7 \%$ of patients had a suprapubic catheter inserted on the tenth postoperative day for up to 2 weeks in a study that entailed prophylactic TVT sling insertion for occult stress incontinence at the time of having vaginal surgery for severe pelvic organ prolapse [3].

Evidence from comparative studies is equally conflicting. On one hand, a study [5] indicated that 7.5\%

Table 5 Complications of TVT sling insertions alongside other gynaecological procedures

\begin{tabular}{|c|c|c|c|}
\hline Complication & All patients $(N=116)$ & Previous continence surgery $(n=13)$ & Major concomitant surgery $(n=80)$ \\
\hline All cases & $22(19.0 \%)$ & $4(30.8 \%)$ & $25(31.3 \%)^{\mathrm{a}}$ \\
\hline Bladder perforation & $1(0.9 \%)$ & $0(0 \%)$ & $0(0 \%)$ \\
\hline Bleeding $\geq 500 \mathrm{ml}$ & $2(1.7 \%)$ & $1(7.7 \%)$ & $1(1.3 \%)$ \\
\hline Blood transfusion & $1(0.9 \%)$ & $0(0 \%)$ & $1(1.3 \%)$ \\
\hline Urinary tract infection & $3(2.6 \%)$ & $1(7.7 \%)$ & $3(3.8 \%)$ \\
\hline Suprapubic infection & $1(0.9 \%)$ & $0(0 \%)$ & $0(0 \%)$ \\
\hline Vaginal infection & $1(0.9 \%)$ & $0(0 \%)$ & $1(1.3 \%)$ \\
\hline Vaginal haematoma & $1(0.9 \%)$ & $1(0.9 \%)$ & $1(1.3 \%)$ \\
\hline Catheter $>7$ days & $11(9.5 \%)$ & $0(0 \%)$ & $6(7.5 \%)$ \\
\hline Tape stretch under general anaesthesia & $7(6.0 \%)$ & $2(15.4 \%)$ & $7(8.8 \%)$ \\
\hline Intermittent self-catheterisation & $2(1.7 \%)$ & $0(0 \%)$ & $1(1.3 \%)$ \\
\hline Tape erosion & $1(0.9 \%)$ & $0(0 \%)$ & $1(1.3 \%)$ \\
\hline Sexual dysfunction & $1(0.9 \%)$ & $0(0 \%)$ & $1(1.3 \%)$ \\
\hline
\end{tabular}

${ }^{\mathrm{a}}$ Significant difference $(p=0.048), \chi^{2}$ test 
Table 6 Outcome at 6 weeks of follow-up

\begin{tabular}{|c|c|c|}
\hline Outcome & Number & Percentage \\
\hline Cure from stress incontinence & 109 & 94 \\
\hline Improved stress incontinence & 5 & 4.3 \\
\hline Failure, repeat TVT sling insertion & 2 & 1.7 \\
\hline De novo overactive bladder symptoms ${ }^{\mathrm{a}}$ & 9 & 8.5 \\
\hline
\end{tabular}

${ }^{a}$ Out of the 106 patients who had pure urodynamic stress incontinence

of patients had voiding difficulties lasting more than 15 days when TVT sling was inserted alongside vaginal hysterectomy and there was no significant increase with concomitant surgery; another showed a significant increase in urinary retention in patients having mesh repair for pelvic organ prolapse with TVT sling insertion only up to 7 days after surgery, but no significant difference beyond that [10]. On the other hand, patients having TVT sling insertion alongside vaginal hysterectomy with or without posterior vaginal wall repair required a significantly longer duration of catheterisation than TVT sling insertion alone [5], and another study reported more cases of transient urinary retention in patients having TVT sling insertion alongside vaginal surgery for pelvic organ prolapse [11]. It is worth bearing in mind that neither this series nor published studies were powered to assess the impact of concomitant surgery on retention of urine following TVT sling insertion.

Seven patients $(6 \%)$ required stretching the sling under general anaesthesia. No such practice was described in the randomised controlled trial that compared TVT sling to Burch colposuspension [16], and it is to be noted that sling stretching was carried out only in three of the five hospitals included in this study, which again shows variation in bladder management. Stretching was needed in $10.4 \%$ of cases in one study that included patients having vaginal surgery for pelvic organ prolapse or laparoscopic-assisted vaginal hysterectomy, though no information was provided about the type of anaesthesia used for sling stretching [6]. Sling incision was performed in 2 out of $26(7.7 \%)$ patients after TVT sling insertion alongside mesh repair for pelvic organ prolapse [10]. Notwithstanding the variation in bladder management and conflicting evidence, the overall impression of the results of this series as well as available evidence is that voiding dysfunction can be a temporary problem following TVT sling insertion alongside concomitant gynaecological surgery, but only a minority of patients may need sling stretching/incision or long-term intermittent self-catheterisation.

It is worth looking at voiding dysfunction when concomitant surgery was carried out without TVT sling insertion. It was difficult to do this in all five hospitals included in this series, in view of the distance and number of cases involved. Looking at published studies is fraught with difficulties due to considerable variation in bladder management as well as the criteria used to define voiding dysfunction [20]. In addition, there are no studies comparing non-continence gynaecological surgery with or without TVT sling insertion. One study [21] reported voiding dysfunction in $8.7 \% 72 \mathrm{~h}$ after vaginal surgery for pelvic organ prolapse, dropping to $1.1 \%$ after 6 days, with no patients needing intermittent self-catheterisation at 2 months of follow-up. Eight per cent of the patients included in the study had Kelley's sutures (bladder neck buttressing), as a continence procedure, carried out at the same time. The study showed large cystocele (grade $\geq 3$ ), excessive intraoperative blood loss and levator plication, in addition to Kelley's sutures (bladder neck buttressing), to be independent predictors of short-term retention of urine. With respect to more general gynaecological surgery, one study reported an overall retention of urine incidence of $9.2 \%$ following a variety of gynaecological procedures, including surgery for pelvic organ prolapse in $10.2 \%$ of cases [20]. This was based on ultrasound post-void bladder volume exceeding preoperative bladder capacity, which in turn was estimated by adding voided volume at spontaneous uroflowmetry to ultrasound post-voided bladder volume. The highest rate was following laparotomy and abdominal hysterectomy, reaching $55.6 \%$ and $13.7 \%$, respectively. Regarding vaginal vault surgery, one study reported $15.6 \%$ and $8.3 \%$ urinary retention beyond 5 days following abdominal sacrocolpopexy and sacropsinous fixation, respectively [22] and another reported spontaneous voiding within 7 days in $75 \%$ patients following sacrospinous fixation, with seven patients $(11.1 \%)$ continuing to have voiding dysfunction at follow-up which resolved within 3 months.

Whilst these studies report higher as well as lower rates of early voiding dysfunction than encountered in this series, long-term voiding dysfunction was lower than those encountered in this series. However, these studies did not focus on continence surgery. It is pertinent to bear in mind that voiding dysfunction can be affected by a multitude of factors, including patient age, nature of surgery, type of anaesthesia, postoperative pain and its management, hydration and ambulation even in surgery not involving the abdomen or the pelvis [23]. This is not to undervalue the observation that inserting TVT slings alongside other gynaecological operations may increase the incidence of early voiding dysfunction as this can potentially have longterm complications [24], but rather shows the complexity of assessing the possible link, which in turn highlights the need for further studies into this area as part of assessing the safety and effectiveness of this combined approach.

Nine out of the 106 patients (8.5\%) who had pure urodynamic stress incontinence prior to TVT sling insertion reported new-onset overactive bladder symptoms at 6 weeks 
of follow-up. The randomised controlled trial that compared TVT sling to Burch colposuspension [16] and one of the studies that reported TVT sling insertion alongside other gynaecological surgery [7] gave no information about overactive bladder symptoms or the incidence of de novo detrusor overactivity. The lowest reported rate was $1.7 \%$ in a study that looked at insertions alongside vaginal surgery for pelvic organ prolapse [8]. One study reported an incidence of $6 \%$ in a mixed group of patients having TVT sling insertion with as well as without concomitant vaginal surgery for pelvic organ prolapse and was not significantly different between the two groups [11]. The incidence was $6.7 \%$ in one study that included patients having laparoscopic-assisted vaginal hysterectomy [14] and 8\% after TVT sling insertion alongside vaginal surgery for pelvic organ prolapse [13]. The highest rate was $13.3 \%$ following prophylactic TVT sling insertion for occult stress incontinence at the time of having vaginal surgery for severe pelvic organ prolapse [3]. The rate found in this study is therefore within the reported range in published studies.

Ninety-four per cent of patients were dry at 6 weeks, and two patients $(1.7 \%)$ had failures that required repeat sling insertion. Whilst this is better than the randomised trial that compared TVT sling to Burch colposuspension [16], the outcome in this series was based on subjective evaluation at 6 weeks of follow-up rather than urodynamic assessment at 6 months, which was the case in the randomised trial. It is also higher than in other studies that reported objective outcome at longer duration of follow-up. In one study that involved 45 patients having vaginal surgery for pelvic organ prolapse at the time of sling insertion [9], the objective cure rate was $42.5 \%$, on the basis of urodynamic assessment at 1-year follow-up, bearing in mind that only $40(88.9 \%)$ patients had this assessment. Another study [13] reported an objective failure rate of $8 \%$ at a median followup duration of 25 months. In one study [3], 10\% of patients had positive stress test 3-6 months after surgery, though none reported stress incontinence. This study, however, entailed prophylactic TVT sling insertion for occult stress incontinence at the time of having vaginal surgery for severe pelvic organ prolapse. On the other hand, the cure rate in this series matches the rates in most of the published studies that looked at TVT sling insertions alongside vaginal surgery for pelvic organ prolapse and vaginal as well as laparoscopic-assisted vaginal hysterectomy [4-6, 8, $12,14]$.

All major abdominal procedures were performed under general anaesthesia before the insertion of the TVT sling. However, there was considerable variation in surgical as well as anaesthetic technique with all other procedures. This was the case even in the same hospital and sometimes for the same surgeon, as reported in some of the published reports $[3-8,10-14]$, and simply illustrates the lack of evidence to support one form of anaesthesia or a particular order in doing concomitant surgery over another. Whilst it could be argued that TVT sling prior to vaginal surgery for pelvic organ prolapse may be inappropriate, as prolapse surgery may affect the position, and thus tension, of the tape [11], larger prospective randomised controlled trials will help in clarifying these aspects. This is particularly important to avoid styles like waking patients up for cough test to adjust the tension of the TVT sling after general anaesthesia for major surgery.

The results of this series should be considered in light of its limitations. There was no control arm as it was not easy to identify patients who had their surgery on two sittings. Several published studies did not include a control arm [4, $6-8,13]$, and even the ones that included a control arm did not actually have a proper one. Some compared patients having TVT sling insertion alongside concomitant surgery to those having TVT sling insertion alone [3, 9, 11, 12, 14], and one compared them to those having the concomitant surgery without TVT sling insertion [10]. A proper comparison would be comparing single-sitting versus twositting approach to those in need of TVT sling insertion alongside other gynaecological procedures. The series, as well most published studies [3-14], only looked at the safety and effectiveness of the TVT sling complications and outcome rather than those of the other gynaecological procedure(s) carried out at the same time. The other side of the comparison, for example looking at hysterectomy alone versus hysterectomy and TVT sling insertion, is also needed to complete the picture. Only one study looked at mesh repair for pelvic organ prolapse with or without concomitant tension-free vaginal tape sling insertion [10].

Other limitations of the study include its retrospective nature and the fact that it only provided short-term outcome. These limitations also mark available studies. Some studies were retrospective $[10,12]$ and none of the published prospective studies were randomised $[4,5,8,11$, 13]. It is true that most studies included long follow-up ranging from 1 year $[4,6,7,13]$ to 2 years [12] and 3 years [11]. However, this longer term follow-up did not include all patients [6] and over a third of patients had $<6$ months of follow-up in one study [12], and long-term data were amalgamated together for those who had TVT sling insertion alone and those who had the insertion alongside other gynaecological operations in another [11]. The series here focused on perioperative complications and subjective short-term outcome, usually when patients are discharged to their general practitioners at British hospitals. This reduces the limitation of the short-term nature of the follow-up.

The study relied on subjective outcome and entailed a relatively small number of cases from each hospital. 
Several published studies relied on subjective outcome [3, $11,12]$. Some of the studies that included objective assessment did not include a full urodynamic assessment $[4,6,8,13]$, and not all patients had full urodynamic assessment when it was included [7, 9, 10]. Although the number of cases included in this series exceeded that required by power calculation, cases came from a number of hospitals. This meant that small numbers were obtained from individual hospitals, let alone individual consultants. However, this reflects the frequency with which such concomitant surgery was carried out at the same time as having TVT sling insertion. Whilst it is difficult to identify how many cases had surgery in separate sittings, addressing this area may encourage others to look at it further. All these features underline the limitations of available evidence in this area.

Despite these limitations, the series has a number of features not matched in published studies. It represents the largest series looking at TVT sling alongside concomitant gynaecological surgery. Numbers included in published studies ranged from 26 [10] and 28 [12] to 75 [11, 13]. The series was based on power calculation, which was not carried out in any of the published studies [3, 4, 6-14]. The series included the full range of minor and major abdominal, vaginal and laparoscopic operations for prolapse as well as other gynaecological problems carried out alongside TVT sling, providing a real-life account of current UK practice. It involved those who had previous continence and prolapse surgery as well as those with mixed incontinence.

Although the grade of evidence provided in this series is reduced by its limitations, it is the best available evidence that reflects real life in British Hospitals. It provides reassurance that concomitant surgery alongside TVT sling insertion does not increase its risk or reduce its effectiveness, apart from a possible rise in short-term retention of urine. Its main value, however, is showing the significant variation in practice, therefore highlighting the need for more and better research into this area. Such a detailed look would require looking at far greater number of hospitals. Whilst the availability of a national register (www.BSUG. net) should enhance access to larger cases, all relevant factors are better addressed in a randomised study with provision for long-term follow-up.

\section{Conclusion}

From examining the notes of the 116 cases included in this study, it possible to conclude that having TVT sling insertion for urodynamic stress incontinence alongside other gynaecological operations does not increase the incidence of operative or early postoperative complications associated with TVT sling insertion or reduce its subjective short-term effectiveness. It is likely that concomitant surgery effects an increase in short-term voiding dysfunction, though this needs further evaluation. A considerable variation in anaesthetic as well as surgical technique was observed, reflecting the lack of evidence to support one approach over another and calling for randomised studies to inform best practice.

Acknowledgment The authors are grateful to Lorna Keyes and Lesley Stroud at Singleton Hospital, Julie Jones at Wrexham Maelor Hospital, Jennifer Allo, Amanda Kenchington and Martin Poore at the Royal Bournemouth Hospital, Elizabeth Cummins at North Devon District Hospital and Suan Phipps and Charlotte Mitchell at Yeovil District Hospital for their help in getting patient notes.

Declaration of interest The authors report no conflicts of interest. The authors alone are responsible for the content and writing of the paper.

\section{References}

1. Chaikin DC, Groutz A, Blaivas JG (2000) Predicting the need for anti-incontinence surgery in women undergoing repair of severe urogenital prolapse. J Urol 163:531-534

2. Sze EH, Milkos JR, Karram MM (1996) Voiding after Burch colposuspension and effects of concomitant pelvic surgery: correlation with pre-operative voiding mechanism. Obstet Gynecol 88:564-567

3. Gordon D, Gold RS, Pauzner D, Lessing JB, Groutz A (2001) Combined genitourinary prolapse repair and prophylactic tensionfree vaginal tape in women with severe prolapse and occult stress urinary incontinence: preliminary results. Urol 58:547-550

4. Jomaa M (2001) Combined tension-free vaginal tape and prolapse repair under local anaesthesia in patients with symptoms of both urinary incontinence and prolapse. Gynecol Obstet Investig $51: 184-186$

5. Darai E, Jeffry L, Deval A, Birsan A, Kadoch O, Soriano D (2002) Results of tension-free vaginal tape in patients with or without vaginal hysterectomy. Eur J Obstet Gynecol 103:163-167

6. Patrol LM (2002) Efficacy of tension-free vaginal tape with other pelvic reconstructive surgery. J Obstet Gynaecol 186:1292-1298

7. Huang KH, Kung FT, Liang HM, Huang LY, Chang SY (2003) Concomitant surgery with tension-free vaginal tape. Act Obstet Gynecol Scand 82:948-953

8. Lo TS, Chang TC, Chao AS, Chou HH, Tseng LH, Liang CC (2003) Tension-free vaginal tape procedure on genuine stress incontinent women with coexisting genital prolapse. Act Obstet Gynecol Scand 82:1049-1053

9. Pang MW, Chan LW, Yip SK (2003) One-year urodynamic outcome and quality of life in patients with concomitant tensionfree vaginal tape during pelvic floor reconstruction surgery for genitourinary prolapse and urodynamic stress incontinence. Int Urogynecol J 14:256-260

10. de Tayrac R, Gervaise A, Chauveaud-Lambling A, Fernandez H (2004) Combined genital prolapse repair reinforced with a polypropylene mesh and tension-free vaginal tape in women with genital prolapse and stress incontinence: a retrospective casecontrol study with short-term follow up. Act Obstet Gynecol Scand 83:950-954 
11. Meltomaa S, Backman T, Haarala M (2004) Concomitant vaginal surgery did not affect the outcome of the tension-free vaginal tape operation during a prospective 3-year follow up study. J Urol 172:222-226

12. Yalcin O, Isikoglu M, Beji NK (2004) Results of TVT operations alone and combined with other vaginal surgical procedures. Arch Gynecol Obstet 269:96-98

13. Huang KH, Kung FT, Liang HM, Chen CW, Chang SY, Hwang LL (2005) Concomitant pelvic organ prolapse surgery with TVT procedure. Int Urogynecol J 17:60-65

14. Lin YH, Llang CC, Soong YK, Soong YK, Chang SD, Chang YL (2005) Concomitant tension-free vaginal tape for urinary incontinence during laparoscopic hysterectomy. Aust NZ J Obstet Gynaecol 45:304-307

15. National Institute for Clinical Excellence (2003) Guidance of the use of tension-free vaginal tape (Gynecare TVT) for stress incontinence, Technology Appraisal Guidance No. 56. National Institute for Clinical Excellence, London, UK

16. Ward K, Hilton P, UK and Ireland TVT Trial Group (2002) Prospective multicentre randomised trial of tension-free vaginal tape and colposuspension as primary treatment for stress incontinence. BMJ 325:67-73

17. Ulmsten U, Henriksson L, Johnson P, Varhos G (1996) An ambulatory surgical procedure under local anaesthesia for treatment of female urinary incontinence. Int Urogynecol J 7:81-86
18. Haab F, Sananes S, Amarenco G, Ciofu C, Uzan S, Gattegno B, Thibault P (2001) Results of the tension-free vaginal tape procedure for the treatment of type II stress urinary incontinence at a minimum follow-up of 1 year. J Urol 165:159-162

19. Mazouni C, Karsenty G, Bretelle F, Bladou F, Gamerre M, Serment G (2004) Urinary complications and sexual function after the tension-free vaginal tape procedure. Act Obstet Gynecol Scand 83:955-961

20. Bødker B, Lose G (2003) Postoperative urinary retention in gynecologic patients. Int Urogynecol J 14:94-97

21. Hakvoort RA, Dijkgraaf MG, Burger MP, Emanuel MH, Roovers JP (2009) Predicting short-term urinary retention after vaginal prolapse surgery. Neurourol Urodyn 28:225-228

22. Demirci F, Ozdemir I, Somunkiran A, Topuz S, Iyibozkurt C, Duras Doyran G, Kemik Gul O, Gul B (2007) Perioperative complications in abdominal sacrocolpopexy and vaginal sacrospinous ligament fixation procedures. Int Urogynecol J 18:257-261

23. Tammela T, Kontturi M, Lukkarinen O (1986) Postoperative urinary retention. I. Incidence and predisposing factors. Scand J Urol Nephrol 20:197-201

24. Tammela T, Kontturi M, Lukkarinen O (1986) Postoperative urinary retention. II. Micturition problems after the first catheterization. Scand J Urol Nephrol 20:257-260 